\title{
Septic theta function identities in Ramanujan's lost notebook
}

by

\author{
Seung Hwan Son (Cambridge, MA)
}

1. Introduction. Ramanujan found many theorems on theta functions, and recorded his results in his notebooks and his lost notebook. The goal of this article is to prove a very unusual set of related theta function identities found in his lost notebook.

As usual, set

$$
(a ; q)_{\infty}:=\prod_{k=0}^{\infty}\left(1-a q^{k}\right), \quad|q|<1 .
$$

Ramanujan's symmetric theta function $f(a, b)$ is defined by

$$
f(a, b):=\sum_{k=-\infty}^{\infty} a^{k(k+1) / 2} b^{k(k-1) / 2}, \quad|a b|<1,
$$

which has the same generality as the classical theta functions $\left\{\theta_{i}(z, q)\right\}_{i=1}^{4}$ in $[8$, pp. 463, 464]. After Ramanujan, define the theta function $\varphi(q)$ by

$$
\varphi(q):=f(q, q)=\sum_{k=-\infty}^{\infty} q^{k^{2}} .
$$

If $q=\exp (-\pi \sqrt{n})$, for some positive rational number $n$, the RamanujanWeber class invariant $G_{n}$ is defined by

$$
G_{n}:=2^{-1 / 4} q^{-1 / 24}\left(-q ; q^{2}\right)_{\infty} .
$$

In his notebooks, Ramanujan presented many values of class invariants which he had evaluated. Using Kronecker's limit formula, modular equations, and class field theory, Berndt, Chan and Zhang [5] verified many of these values which had not been previously determined.

2000 Mathematics Subject Classification: Primary 33C05; Secondary 05A30, 11F32, 11R29. 
On page 206 in his lost notebook [6], Ramanujan recorded the following identities, which we now quote. Let

$$
\frac{\varphi\left(q^{1 / 7}\right)}{\varphi\left(q^{7}\right)}=1+u+v+w
$$

Then

$$
p:=u v w=\frac{8 q^{2}\left(-q ; q^{2}\right)_{\infty}}{\left(-q^{7} ; q^{14}\right)_{\infty}^{7}}
$$

and

$$
\frac{\varphi^{8}(q)}{\varphi^{8}\left(q^{7}\right)}-(2+5 p) \frac{\varphi^{4}(q)}{\varphi^{4}\left(q^{7}\right)}+(1-p)^{3}=0
$$

Furthermore,

$$
u=\left(\frac{\alpha^{2} p}{\beta}\right)^{1 / 7}, \quad v=\left(\frac{\beta^{2} p}{\gamma}\right)^{1 / 7}, \quad w=\left(\frac{\gamma^{2} p}{\alpha}\right)^{1 / 7},
$$

where $\alpha, \beta$ and $\gamma$ are the roots of the equation

$$
\xi^{3}+2 \xi^{2}\left(1+3 p-\frac{\varphi^{4}(q)}{\varphi^{4}\left(q^{7}\right)}\right)+\xi p^{2}(p+4)-p^{4}=0 .
$$

For example,

$$
\varphi\left(e^{-7 \pi \sqrt{7}}\right)=7^{3 / 4} \varphi\left(e^{-\pi \sqrt{7}}\right)\left\{1+(-)^{2 / 7}+(-)^{2 / 7}+(-)^{2 / 7}\right\} .
$$

Although $u, v$ and $w$ are not clearly defined in these claims, they are found in Entry 17(iii) in Chapter 19 of Ramanujan's second notebook [1, p. 303]. By comparison with (1.1) and the identity in the entry, we define

$$
u:=2 q^{1 / 7} \frac{f\left(q^{5}, q^{9}\right)}{\varphi\left(q^{7}\right)}, \quad v:=2 q^{4 / 7} \frac{f\left(q^{3}, q^{11}\right)}{\varphi\left(q^{7}\right)}, \quad w:=2 q^{9 / 7} \frac{f\left(q, q^{13}\right)}{\varphi\left(q^{7}\right)} .
$$

Note that the identities of three expressions on the right side of (1.6) are not given. It is unclear if Ramanujan had explicitly determined them.

Ramanujan might have tried to evaluate the value of the quotient

$$
\varphi\left(e^{-\pi \sqrt{7}}\right) / \varphi\left(e^{-7 \pi \sqrt{7}}\right),
$$

by using the identities (1.1)-(1.5). Since $G_{7}=2^{1 / 4}[3$, p. 189], the value of $G_{7^{3}}$ can easily be evaluated by a routine calculation if the quotient (1.8) were known.

In this paper we establish several theorems in order to prove the identities (1.2)-(1.5). We employ modular equations of degree seven, the Jacobi triple product identity, several Lambert series identities and a new product formula for theta functions.

2. Preliminary results. We need the following Jacobi triple product identity. 
Theorem 2.1. For $|a b|<1$, we have

$$
f(a, b)=(-a ; a b)_{\infty}(-b ; a b)_{\infty}(a b ; a b)_{\infty} .
$$

For a proof, see [1, p. 35].

After Ramanujan, we define

$$
f(-q):=f\left(-q,-q^{2}\right) \quad \text { and } \quad \chi(q):=\left(-q ; q^{2}\right)_{\infty} .
$$

By the Jacobi triple product identity, one can prove the identity

$$
f(-q)=(q ; q)_{\infty}
$$

which is Euler's pentagonal number theorem.

To simplify certain theta function products which may be called the Ramanujan products, we shall employ the following general product formula established in [7].

TheOREm 2.2. Let $|a b|<1$, let $j, k$ be arbitrary integers with $(j, k) \not \equiv$ $(0,0)(\bmod p)$, where $p$ is an odd prime, let $\zeta:=\exp (2 \pi i / p)$, and let $s$ be a solution of

$$
(j+k) x+j \equiv 0(\bmod p) \quad \text { and } \quad 0 \leq x<p .
$$

Then

$$
\begin{aligned}
& \prod_{n=1}^{p} f\left(\zeta^{j n} a, \zeta^{k n} b\right) \\
& \quad= \begin{cases}\frac{f^{p}\left(a^{s+1} b^{s}, a^{p-s-1} b^{p-s}\right) f\left(a^{p}, b^{p}\right)}{f\left(a^{p(s+1)} b^{p s}, a^{p(p-s-1)} b^{p(p-s)}\right)} & \text { if } j+k \not \equiv 0(\bmod p), \\
f^{p}(-a b) \frac{f\left(a^{p}, b^{p}\right)}{f\left(-a^{p} b^{p}\right)} & \text { if } j+k \equiv 0(\bmod p) .\end{cases}
\end{aligned}
$$

In what follows, the following three Lambert series are needed.

Theorem 2.3. For $|q|<1, \varphi(q)$ satisfies the following identities:

$$
\begin{aligned}
& \varphi^{4}(q)=1+8 \sum_{k=1}^{\infty} \frac{k q^{k}}{1+(-q)^{k}}, \\
& \varphi^{6}(q)=1-4 \sum_{k=0}^{\infty} \frac{(-1)^{k}(2 k+1)^{2} q^{2 k+1}}{1-q^{2 k+1}}+16 \sum_{k=1}^{\infty} \frac{k^{2} q^{k}}{1+q^{2 k}} \\
& \varphi^{8}(q)=1+16 \sum_{k=1}^{\infty} \frac{k^{3} q^{k}}{1-(-q)^{k}} .
\end{aligned}
$$

For proofs of (i)-(iii), see Entry 8(ii) of [1, p. 114], Entry 2 of [4] and Examples (i) of [1, p. 139], respectively. 
3. Modular equations of degree seven. Ramanujan recorded many modular equations [1, Chaps. 18-21], [2, Chap. 25]. To briefly define a modular equation, we first put, as usual,

$$
(a)_{k}:=\frac{\Gamma(a+k)}{\Gamma(a)} .
$$

A modular equation of degree 7 [1, pp. 213, 214] is an equation relating $\alpha$ and $\beta$ that is induced by

$$
7 \cdot \frac{{ }_{2} F_{1}\left(\frac{1}{2}, \frac{1}{2} ; 1 ; 1-\alpha\right)}{{ }_{2} F_{1}\left(\frac{1}{2}, \frac{1}{2} ; 1 ; \alpha\right)}=\frac{{ }_{2} F_{1}\left(\frac{1}{2}, \frac{1}{2} ; 1 ; 1-\beta\right)}{{ }_{2} F_{1}\left(\frac{1}{2}, \frac{1}{2} ; 1 ; \beta\right)},
$$

where

$$
{ }_{2} F_{1}(a, b ; c ; x):=\sum_{k=0}^{\infty} \frac{(a)_{k}(b)_{k}}{(c)_{k} k !} x^{k}, \quad|x|<1 .
$$

The multiplier $m$ is defined by

$$
m:=\frac{{ }_{2} F_{1}\left(\frac{1}{2}, \frac{1}{2} ; 1 ; \alpha\right)}{{ }_{2} F_{1}\left(\frac{1}{2}, \frac{1}{2} ; 1 ; \beta\right)} .
$$

Let

$$
z_{1}:={ }_{2} F_{1}\left(\frac{1}{2}, \frac{1}{2} ; 1 ; \alpha\right) \quad \text { and } \quad z_{7}:={ }_{2} F_{1}\left(\frac{1}{2}, \frac{1}{2} ; 1 ; \beta\right) .
$$

Then by the definition of a modular equation of degree 7 ,

$$
q^{7}=\exp \left(-7 \pi \frac{{ }_{2} F_{1}\left(\frac{1}{2}, \frac{1}{2} ; 1 ; 1-\alpha\right)}{{ }_{2} F_{1}\left(\frac{1}{2}, \frac{1}{2} ; 1 ; \alpha\right)}\right)=\exp \left(-\pi \frac{{ }_{2} F_{1}\left(\frac{1}{2}, \frac{1}{2} ; 1 ; 1-\beta\right)}{{ }_{2} F_{1}\left(\frac{1}{2}, \frac{1}{2} ; 1 ; \beta\right)}\right) .
$$

By the catalogue of theta function evaluations in [1, pp. 122, 124], we find the following identities.

Lemma 3.1. For $|q|<1$,

$$
\begin{aligned}
\varphi(q) & =\sqrt{z_{1}}, \\
\varphi\left(q^{7}\right) & =\sqrt{z_{7}}, \\
\chi(q) & =2^{1 / 6} q^{1 / 24}(\alpha(1-\alpha))^{-1 / 24}, \\
\chi\left(q^{7}\right) & =2^{1 / 6} q^{7 / 24}(\beta(1-\beta))^{-1 / 24} .
\end{aligned}
$$

For proofs of (3.1)-(3.4), see [1, pp. 122, 124].

Thus,

$$
m=\varphi^{2}(q) / \varphi^{2}\left(q^{7}\right) .
$$

Lemma 3.2. For $|q|<1$,

$$
1-4 \sum_{k=0}^{\infty} \frac{(-1)^{k}(2 k+1)^{2}}{q^{-(2 k+1)}-1}=\varphi^{6}(q)(1-\alpha) .
$$

For a proof, use Entry 17(vii) of [1, p. 138] and (3.1). 
Theorem 3.3. For $t:=(\alpha \beta)^{1 / 8}$, we find that

$$
\begin{gathered}
((1-\alpha)(1-\beta))^{1 / 8}=1-t, \\
m-\frac{7}{m}=-(1-2 t)^{3}-5(1-2 t), \\
\frac{\alpha}{t}-\frac{1-\alpha}{1-t}=\frac{7}{m}(1-t(1-t)) .
\end{gathered}
$$

For a proof of (3.6), see Entry 19(i) of [1, p. 314]. By (3.6) and (viii) and (iii) in Entry 19 of [1, pp. 314, 315], we deduce (3.7) and (3.8), respectively.

Throughout this paper, we use the notation $m, \alpha, \beta, z_{1}, z_{7}$ and $t$ that are defined in this section.

\section{Proofs of the septic identities}

Lemma 4.1. For $|q|<1$ and $p$ in (1.2),

$$
p=\frac{8 q^{2} \chi(q)}{\chi^{7}\left(q^{7}\right)}=4\left(\frac{\beta^{7}(1-\beta)^{7}}{\alpha(1-\alpha)}\right)^{1 / 24} .
$$

Proof. By the Jacobi triple product identity,

$$
\varphi\left(q^{7}\right)=f\left(q^{7}, q^{7}\right)=\left(-q^{7} ; q^{14}\right)_{\infty}^{2}\left(q^{14} ; q^{14}\right)_{\infty} .
$$

Thus, by (2.1) and the above equality, we find that

$$
\begin{aligned}
p=u v w= & \frac{8 q^{2}}{\varphi^{4}\left(q^{7}\right)}\left(-q ; q^{14}\right)_{\infty}\left(-q^{3} ; q^{14}\right)_{\infty}\left(-q^{5} ; q^{14}\right)_{\infty}\left(-q^{7} ; q^{14}\right)_{\infty}^{2} \\
& \times\left(-q^{9} ; q^{14}\right)_{\infty}\left(-q^{11} ; q^{14}\right)_{\infty}\left(-q^{13} ; q^{14}\right)_{\infty}\left(q^{14} ; q^{14}\right)_{\infty}^{4} .
\end{aligned}
$$

Since

$$
\left(-q ; q^{2}\right)_{\infty}=\prod_{k=1}^{7}\left(-q^{2 k-1} ; q^{14}\right)_{\infty},
$$

we can simplify $p$ so that

$$
p=\frac{8 q^{2}\left(-q ; q^{2}\right)_{\infty}}{\left(-q^{7} ; q^{14}\right)_{\infty}^{7}}=\frac{8 q^{2} \chi(q)}{\chi^{7}\left(q^{7}\right)},
$$

where $\chi(q)$ is defined in (2.2). Using (3.3) and (3.4), we complete the proof of (4.1).

Note that (4.2) establishes (1.2).

Lemma 4.2. For $|q|<1$,

$$
1-2 t=\frac{1-p}{m} .
$$

Proof. By using Lemma 4.1 and (3.6) in Entry 19(ii) of [1, p. 314], we complete the proof.

We are now ready to prove (1.3). 
Theorem 4.3. Equality (1.3) is valid.

Proof. By (3.7) and Lemma 4.2, we find that

$$
m-\frac{7}{m}=-\left(\frac{1-p}{m}\right)^{3}-5\left(\frac{1-p}{m}\right) .
$$

Multiplying both sides by $m^{3}$, we obtain

$$
m^{4}-(2+5 p) m^{2}+(1-p)^{3}=0
$$

Thus, by (3.5), we complete the proof.

Lemma 4.4. For $|q|<1$,

$$
\varphi^{4}(q)=1+8 \sum_{k=1}^{\infty} \sum_{n=1}^{\infty}(-1)^{(n-1)(k-1)} k q^{n k} .
$$

Proof. In Theorem 2.3(i), expand the summands in geometric series.

Definition. For convenience, we define the operator $\mathcal{M}_{0}$ which collects the terms whose powers are integers.

Lemma 4.5. For $|q|<1$,

$$
\mathcal{M}_{0}\left(\varphi^{4}\left(q^{1 / 7}\right)\right)=8 \varphi^{4}(q)-7 \varphi^{4}\left(q^{7}\right) .
$$

Proof. Let

$$
U_{k}:=\sum_{n=1}^{\infty}(-1)^{(n-1)(k-1)} k\left(q^{1 / 7}\right)^{n k} \quad \text { and } \quad I_{n}:=\mathcal{M}_{0}\left(\sum_{k \equiv n(\bmod 7)} U_{k}\right) .
$$

By Lemma 4.4,

$$
\mathcal{M}_{0}\left(\varphi^{4}\left(q^{1 / 7}\right)\right)=1+8\left(I_{0}+\ldots+I_{6}\right) .
$$

In $I_{0}$, replacing $k$ by $7 k$, we obtain

$$
I_{0}=7 \sum_{k=1}^{\infty} \sum_{n=1}^{\infty}(-1)^{(n-1)(k-1)} k q^{n k}
$$

For $1 \leq j \leq 6$,

$$
I_{j}=\sum_{k=0}^{\infty} \sum_{n=1}^{\infty}(-1)^{(n-1)(7 k+j-1)}(7 k+j) q^{n(7 k+j)} .
$$

Define

$$
I_{0}^{\prime}:=\sum_{k=1}^{\infty} \sum_{n=1}^{\infty}(-1)^{(n-1)(7 k-1)}(7 k) q^{n(7 k)}
$$


Then, by (4.5) and (4.6),

$$
I_{1}+\ldots+I_{6}+I_{0}^{\prime}=\sum_{k=1}^{\infty} \sum_{n=1}^{\infty}(-1)^{(n-1)(k-1)} k q^{n k} .
$$

Employing (4.4), (4.7) and Lemma 4.4, we deduce that

$$
I_{0}+\ldots+I_{6}=8 \sum_{k=1}^{\infty} \sum_{n=1}^{\infty}(-1)^{(n-1)(k-1)} k q^{n k}-I_{0}^{\prime}=\varphi^{4}(q)-1-I_{0}^{\prime} .
$$

Therefore, by (4.3) and (4.6),

$$
\mathcal{M}_{0}\left(\varphi^{4}\left(q^{1 / 7}\right)\right)=1+8\left(\varphi^{4}(q)-1-I_{0}^{\prime}\right)=8 \varphi^{4}(q)-7 \varphi^{4}\left(q^{7}\right),
$$

by Lemma 4.4 .

Theorem 4.6. For $u, v$ and $w$ defined in (1.7), and $|q|<1$,

$$
u^{3} v+v^{3} w+w^{3} u=-2\left(1+3 p-\frac{\varphi^{4}(q)}{\varphi^{4}\left(q^{7}\right)}\right) .
$$

Proof. By (1.1),

$$
\mathcal{M}_{0}\left(\left(\frac{\varphi\left(q^{1 / 7}\right)}{\varphi\left(q^{7}\right)}\right)^{4}\right)=\mathcal{M}_{0}\left((1+u+v+w)^{4}\right) .
$$

By Lemma 4.5,

$$
\mathcal{M}_{0}\left(\left(\frac{\varphi\left(q^{1 / 7}\right)}{\varphi\left(q^{7}\right)}\right)^{4}\right)=8 \frac{\varphi^{4}(q)}{\varphi^{4}\left(q^{7}\right)}-7 .
$$

By the multinomial expansion and (1.2),

$$
\mathcal{M}_{0}\left((1+u+v+w)^{4}\right)=4\left(u^{3} v+v^{3} w+w^{3} u\right)+24 p+1 .
$$

Using (4.9) and (4.10) in (4.8), we deduce that

$$
u^{3} v+v^{3} w+w^{3} u=2\left(\frac{\varphi^{4}(q)}{\varphi^{4}\left(q^{7}\right)}-3 p-1\right),
$$

which is the desired result.

Lemma 4.7. For $|q|<1$,

$$
16 m^{3} \alpha=8 m^{3}+(p+20) m^{2}+7(3 p+4)(p-1) .
$$

Proof. If we solve the linear equation (3.8) for $\alpha$, then

$$
\alpha=t\left(1-\frac{7}{m}(t-1)\left(t^{2}-t+1\right)\right) .
$$

By Lemma 4.2,

$$
t=\frac{m+p-1}{2 m}
$$


and so

$$
\alpha=\frac{1}{16 m^{5}}\left(8 m^{5}+(8 p+13) m^{4}-14(p-1)^{2} m^{2}-7(p-1)^{4}\right) .
$$

Thus, by the equality

$$
7(p-1)^{4}=7(p-1)\left(m^{4}-(2+5 p) m^{2}\right),
$$

which is a reformulation of (1.3), we find that

$$
\alpha=\frac{1}{16 m^{3}}\left(8 m^{3}+(p+20) m^{2}+7(3 p+4)(p-1)\right),
$$

which is the required result.

THEOREM 4.8. For $|q|<1$,

$$
256 \sum_{k=1}^{\infty} \frac{k^{2} q^{k}}{1+q^{2 k}}=8 \varphi^{6}(q)+(p+20) \varphi^{4}(q) \varphi^{2}\left(q^{7}\right)+7(3 p+4)(p-1) \varphi^{6}\left(q^{7}\right) .
$$

Proof. By Lemma 3.2 and Theorem 2.3(ii),

$$
16 \sum_{k=1}^{\infty} \frac{k^{2} q^{k}}{1+q^{2 k}}=\varphi^{6}(q) \alpha
$$

Applying Lemma 4.7 and (3.5), we arrive at

$$
\frac{256}{\varphi^{6}\left(q^{7}\right)} \sum_{k=1}^{\infty} \frac{k^{2} q^{k}}{1+q^{2 k}}=16 m^{3} \alpha=8 m^{3}+(p+20) m^{2}+7(3 p+4)(p-1),
$$

which is equivalent to the desired result.

LEMMA 4.9. For $|q|<1$, $\varphi^{6}(q)=1-4 \sum_{k=0}^{\infty} \sum_{n=1}^{\infty}(-1)^{k}(2 k+1)^{2} q^{n(2 k+1)}+16 \sum_{k=0}^{\infty} \sum_{n=1}^{\infty}(-1)^{k} n^{2} q^{n(2 k+1)}$.

Proof. Applying Theorem 2.3(ii), we deduce that

$$
\sum_{k=0}^{\infty} \frac{(-1)^{k}(2 k+1)^{2} q^{2 k+1}}{1-q^{2 k+1}}=\sum_{k=0}^{\infty} \sum_{n=1}^{\infty}(-1)^{k}(2 k+1)^{2} q^{n(2 k+1)}
$$

and

$$
\sum_{n=1}^{\infty} \frac{n^{2} q^{n}}{1+q^{2 n}}=\sum_{k=0}^{\infty} \sum_{n=1}^{\infty}(-1)^{k} n^{2} q^{n(2 k+1)}
$$

after expanding the summands in geometric series. Substituting (4.11) and (4.12) in Theorem 2.3(ii), we complete the proof.

LEMMA 4.10. For $|q|<1$,

$$
\mathcal{M}_{0}\left(\varphi^{6}\left(q^{1 / 7}\right)\right)=6(p+20) \varphi^{4}(q) \varphi^{2}\left(q^{7}\right)+7\left(18 p^{2}-6 p-17\right) \varphi^{6}\left(q^{7}\right) .
$$


Proof. Let

$$
U_{k}:=\sum_{n=1}^{\infty}(-1)^{k}(2 k+1)^{2}\left(q^{1 / 7}\right)^{n(2 k+1)}, \quad V_{k}:=\sum_{n=1}^{\infty}(-1)^{k} n^{2}\left(q^{1 / 7}\right)^{n(2 k+1)} .
$$

For convenience, we define

$$
I_{n}:=\mathcal{M}_{0}\left(\sum_{k \equiv n(\bmod 7)} U_{k}\right) \text { and } J_{n}:=\mathcal{M}_{0}\left(\sum_{k \equiv 0(\bmod 7)} V_{k}\right) .
$$

By Lemma 4.9,

$$
\mathcal{M}_{0}\left(\varphi^{6}\left(q^{1 / 7}\right)\right)=1-4\left(I_{0}+\ldots+I_{6}\right)+16\left(J_{0}+\ldots+J_{6}\right) .
$$

As $2(7 k+j)+1 \equiv 0(\bmod 7)$ for $j=3$,

$$
I_{3}=-7^{2} \sum_{k=0}^{\infty} \sum_{n=1}^{\infty}(-1)^{k}(2 k+1)^{2} q^{n(2 k+1)} .
$$

For $j \neq 3$,

$$
I_{j}=\sum_{k=0}^{\infty} \sum_{n=1}^{\infty}(-1)^{7 k+j}(2(7 k+j)+1)^{2} q^{n(2(7 k+j)+1)} .
$$

Define

$$
I_{3}^{\prime}:=-7^{2} \sum_{k=0}^{\infty} \sum_{n=1}^{\infty}(-1)^{k}(2 k+1)^{2}\left(q^{7}\right)^{n(2 k+1)} .
$$

Thus, by (4.15) and (4.16),

$$
I_{0}+I_{1}+I_{2}+I_{3}^{\prime}+I_{4}+I_{5}+I_{6}=\sum_{k=0}^{\infty} \sum_{n=1}^{\infty}(-1)^{k}(2 k+1)^{2} q^{n(2 k+1)} .
$$

Then we arrive at

$$
I_{0}+\ldots+I_{6}=-48 \sum_{k=0}^{\infty} \sum_{n=1}^{\infty}(-1)^{k}(2 k+1)^{2} q^{n(2 k+1)}-I_{3}^{\prime},
$$

by (4.14) and (4.17).

Similarly,

$$
J_{0}+\ldots+J_{6}=48 \sum_{k=0}^{\infty} \sum_{n=1}^{\infty}(-1)^{k} n^{2} q^{n(2 k+1)}-J_{3}^{\prime},
$$

where

$$
J_{3}^{\prime}:=-7^{2} \sum_{k=0}^{\infty} \sum_{n=1}^{\infty}(-1)^{k} n^{2}\left(q^{7}\right)^{n(2 k+1)} .
$$


Therefore, by (4.13) and (4.18)-(4.20),

$$
\begin{aligned}
\mathcal{M}_{0}\left(\varphi^{6}\left(q^{1 / 7}\right)\right)= & +4 \cdot 48 \sum_{k=0}^{\infty} \sum_{n=1}^{\infty}(-1)^{k}(2 k+1)^{2} q^{n(2 k+1)}+4 I_{3}^{\prime} \\
& +16 \cdot 48 \sum_{k=0}^{\infty} \sum_{n=1}^{\infty}(-1)^{k} n^{2} q^{n(2 k+1)}-16 J_{3}^{\prime} .
\end{aligned}
$$

By Lemma 4.9, we deduce that

$$
\begin{aligned}
& \mathcal{M}_{0}\left(\varphi^{6}\left(q^{1 / 7}\right)\right) \\
& \quad=49 \varphi^{6}\left(q^{7}\right)-48 \varphi^{6}(q)+2 \cdot 16 \cdot 48 \sum_{k=0}^{\infty} \sum_{n=1}^{\infty}(-1)^{k} n^{2} q^{n(2 k+1)} .
\end{aligned}
$$

Employing Theorem 4.8, we find that

$$
\begin{aligned}
2 & \cdot 16 \cdot 48 \sum_{k=0}^{\infty} \sum_{n=1}^{\infty}(-1)^{k} n^{2} q^{n(2 k+1)} \\
& =6 \cdot 256 \sum_{n=1}^{\infty} \frac{n^{2} q^{n}}{1+q^{2 n}} \\
& =6\left(8 \varphi^{6}(q)+(p+20) \varphi^{4}(q) \varphi^{2}\left(q^{7}\right)+7(3 p+4)(p-1) \varphi^{6}\left(q^{7}\right)\right) .
\end{aligned}
$$

Thus, by substituting (4.22) into (4.21), we complete the proof.

Lemma 4.11. For $|q|<1$,

$$
\varphi^{8}(q)=1+16 \sum_{k=1}^{\infty} \sum_{n=1}^{\infty}(-1)^{(n-1) k} k^{3} q^{n k} .
$$

Proof. In Theorem 2.3(iii), expand the summands in geometric series.

Lemma 4.12. For $|q|<1$,

$$
\mathcal{M}_{0}\left(\varphi^{8}\left(q^{1 / 7}\right)\right)=344 \varphi^{8}(q)-343 \varphi^{8}\left(q^{7}\right)
$$

Proof. Let

$$
U_{k}:=\sum_{n=1}^{\infty}(-1)^{(n-1) k} k^{3}\left(q^{1 / 7}\right)^{n k} \text { and } I_{n}:=\mathcal{M}_{0}\left(\sum_{k \equiv n(\bmod 7)} U_{k}\right) .
$$

By Lemma 4.11,

$$
\mathcal{M}_{0}\left(\varphi^{8}\left(q^{1 / 7}\right)\right)=1+16\left(I_{0}+\ldots+I_{6}\right) .
$$

In $I_{0}$, replacing $k$ by $7 k$, we obtain

$$
I_{0}=7^{3} \sum_{k=1}^{\infty} \sum_{n=1}^{\infty}(-1)^{(n-1) k} k^{3} q^{n k}
$$


For $1 \leq j \leq 6$,

$$
I_{j}=\sum_{k=0}^{\infty} \sum_{n=1}^{\infty}(-1)^{(n-1)(7 k+j)}(7 k+j)^{3} q^{n(7 k+j)} .
$$

Define

$$
I_{0}^{\prime}:=7^{3} \sum_{k=1}^{\infty} \sum_{n=1}^{\infty}(-1)^{(n-1) k} k^{3}\left(q^{7}\right)^{n k} .
$$

Thus, by (4.25) and (4.26),

$$
I_{1}+\ldots+I_{6}+I_{0}^{\prime}=\sum_{k=1}^{\infty} \sum_{n=1}^{\infty}(-1)^{(n-1) k} k^{3} q^{n k} .
$$

Using (4.24) and (4.27), we find that

$$
I_{0}+\ldots+I_{6}=344 \sum_{k=1}^{\infty} \sum_{n=1}^{\infty}(-1)^{(n-1) k} k^{3} q^{n k}-I_{0}^{\prime} .
$$

Hence, by (4.23), (4.27) and (4.26),

$$
\begin{aligned}
\mathcal{M}_{0}\left(\varphi^{8}\left(q^{1 / 7}\right)\right)= & 344\left(1+16 \sum_{k=1}^{\infty} \sum_{n=1}^{\infty}(-1)^{(n-1) k} k^{3} q^{n k}\right) \\
& -343\left(1+16 \sum_{k=1}^{\infty} \sum_{n=1}^{\infty}(-1)^{(n-1) k} k^{3}\left(q^{7}\right)^{n k}\right) \\
= & 344 \varphi^{8}(q)-343 \varphi^{8}\left(q^{7}\right),
\end{aligned}
$$

by Lemma 4.11. This completes the proof.

Definition. For convenience, we define $X:=u^{2} v^{3}+v^{2} w^{3}+w^{2} u^{3}, \quad Y:=u v^{5}+v w^{5}+w u^{5}, \quad Z:=u^{7}+v^{7}+w^{7}$.

Lemma 4.13. For $|q|<1$,

$$
10 X+Y=p\left(m^{2}+6 p+47\right) .
$$

Proof. By (1.1),

$$
\mathcal{M}_{0}\left(\left(\frac{\varphi\left(q^{1 / 7}\right)}{\varphi\left(q^{7}\right)}\right)^{6}\right)=\mathcal{M}_{0}\left((1+u+v+w)^{6}\right) .
$$

By Lemma 4.10,

$$
\mathcal{M}_{0}\left(\left(\frac{\varphi\left(q^{1 / 7}\right)}{\varphi\left(q^{7}\right)}\right)^{6}\right)=6(p+20) m^{2}+7\left(18 p^{2}-6 p-17\right) .
$$

By the multinomial expansion, (1.2) and Theorem 4.6,

$$
\mathcal{M}_{0}\left((1+u+v+w)^{6}\right)=60 X+6 Y+90 p^{2}-120\left(3 p+1-m^{2}\right)+120 p+1 .
$$

Using (4.29) and (4.30) in (4.28), we finish the proof. 
Lemma 4.14. For $|q|<1$,

$$
14 X-7 Y+Z=m^{4}-14(p-1) m^{2}+49 p^{2}-42 p-15 .
$$

Proof. Let $\zeta:=\exp (2 \pi i / 7)$. Replacing $q^{1 / 7}$ by $\zeta q^{1 / 7}$ in (1.1), and then multiplying the seven identities, and recalling the definitions of $u, v$ and $w$ in (1.7), we obtain

$$
\frac{1}{\varphi^{7}\left(q^{7}\right)} \prod_{k=1}^{7} \varphi\left(\zeta q^{1 / 7}\right)=\prod_{k=1}^{7}\left(1+\zeta^{k} u+\zeta^{4 k} v+\zeta^{9 k} w\right) .
$$

By Theorem 2.2 with $a=b=q^{1 / 7}, j=k=1$ and $s=3$,

$$
\frac{1}{\varphi^{7}\left(q^{7}\right)} \prod_{k=1}^{7} \varphi\left(\zeta q^{1 / 7}\right)=\frac{\varphi^{8}(q)}{\varphi^{8}\left(q^{7}\right)} .
$$

Using (1.2) and Theorm 4.6, we find that the right side of (4.31) becomes

$$
\begin{aligned}
Z-14 p\left(3 p+1-m^{2}\right)-7 Y-7 p^{2} & \\
+ & 14 X+14\left(3 p+1-m^{2}\right)+14 p+1 .
\end{aligned}
$$

By (3.5), (4.31)-(4.33), and simplification, we complete the proof.

Lemma 4.15. For $|q|<1$,

$14(2 p+5) X+21 Y+Z=m^{4}+14(p+1) m^{2}+28 p^{3}+105 p^{2}+378 p-15$.

Proof. By (1.1),

$$
\mathcal{M}_{0}\left(\left(\frac{\varphi\left(q^{1 / 7}\right)}{\varphi\left(q^{7}\right)}\right)^{8}\right)=\mathcal{M}_{0}\left((1+u+v+w)^{8}\right) .
$$

By Lemma 4.12 and (1.3),

$$
\begin{aligned}
\mathcal{M}_{0}\left(\left(\frac{\varphi\left(q^{1 / 7}\right)}{\varphi\left(q^{7}\right)}\right)^{8}\right) & =344 m^{4}-343=120 m^{4}+224 m^{4}-343 \\
& =120 m^{4}+224\left((5 p+2) m^{2}+(p-1)^{3}\right)-343
\end{aligned}
$$

By the multinomial expansion, (1.2) and Theorem 4.6,

$$
\begin{aligned}
\mathcal{M}_{0}((1+u+v & \left.+w)^{8}\right) \\
= & 28\left(u^{6} v^{2}+v^{6} w^{2}+w^{6} u^{2}\right) \\
& +280 p X+8 Z+2520 p^{2}-1680 p\left(3 p+1-m^{2}\right) \\
& +168 Y+560 X-560\left(3 p+1-m^{2}\right)+336 p+1 .
\end{aligned}
$$

By Theorem 4.6,

$$
\left(u^{3} v+v^{3} w+w^{3} u\right)^{2}=4\left(3 p+1-m^{2}\right)^{2} .
$$


Thus, by (4.37) and (1.2),

$$
u^{6} v^{2}+v^{6} w^{2}+w^{6} u^{2}=4\left(3 p+1-m^{2}\right)^{2}-2 p X .
$$

By (4.34)-(4.36), (4.38), and simplification, we complete the proof.

Theorem 4.16. For $u, v$ and $w$ defined in (1.7), and $|q|<1$,

$$
\begin{gathered}
u^{2} v^{3}+v^{2} w^{3}+w^{2} u^{3}=p(p+4), \\
u v^{5}+v w^{5}+w u^{5}=p\left(\frac{\varphi^{4}(q)}{\varphi^{4}\left(q^{7}\right)}-4 p+7\right)
\end{gathered}
$$

and

$$
u^{7}+v^{7}+w^{7}=\frac{\varphi^{8}(q)}{\varphi^{8}\left(q^{7}\right)}-7(p-2) \frac{\varphi^{4}(q)}{\varphi^{4}\left(q^{7}\right)}+7 p^{2}-49 p-15 .
$$

Proof. We have a system of equations given by Lemmas 4.13-4.15. By solving this linear system for $X, Y$ and $Z$, we complete the proof.

We are now in a position to prove (1.5).

Theorem 4.17. Equality (1.5) is valid.

Proof. Without loss of generality, one can assume that

$$
\alpha:=u^{3} v, \quad \beta:=v^{3} w, \quad \gamma:=w^{3} u .
$$

By solving the system (4.40) for $u, v$ and $w$, we obtain (1.4). By Theorem 4.6, (4.39) and (1.2), we find that

$$
\alpha+\beta+\gamma=-2\left(1+3 p-m^{2}\right), \quad \alpha \beta+\beta \gamma+\gamma \alpha=p^{2}(p+4), \quad \alpha \beta \gamma=p^{4} .
$$

Thus $\alpha, \beta$ and $\gamma$ are roots of the equation (1.5).

REMARK. Why did Ramanujan not give the explicit identification for the three missing terms in (1.6)? Did Ramanujan know the structure of the quotient (1.8), or did he merely conjecture that $\varphi\left(e^{-7 \pi \sqrt{7}}\right)$ has the expression in the form (1.6)?

The author conjectures that Ramanujan had a result of this form, but he decided not to record it because it was inelegant. Unfortunately, the author was unable to prove any theorem of this type. If Ramanujan had found an explicit representation of the quotient (1.8), he would have known the value of the class invariant $G_{343}=G_{7^{3}}$, which would have been a new result, which up to the present day has not been explicitly calculated.

Acknowledgments. The author would like to express deep appreciation to Professor Bruce C. Berndt for his warm encouragement and invaluable suggestions. 


\section{References}

[1] B. C. Berndt, Ramanujan's Notebooks, Part III, Springer, New York, 1991.

[2] -, Ramanujan's Notebooks, Part IV, Springer, New York, 1994.

[3] -, Ramanujan's Notebooks, Part V, Springer, New York, 1998.

[4] - Fragments by Ramanujan on Lambert series, in: Number Theory and Its Applications, K. Györy and S. Kanemitsu (eds.), Kluwer, Dordrecht, 1999, 35-49.

[5] B. C. Berndt, H. H. Chan and L.-C. Zhang, Ramanujan's class invariants, Kronecker's limit formula, and modular equations, Trans. Amer. Math. Soc. 349 (1997), 2125-2173.

[6] S. Ramanujan, The Lost Notebook and Other Unpublished Papers, Narosa, New Delhi, 1988.

[7] S. H. Son, Some theta function identities related to the Rogers-Ramanujan continued fraction, Proc. Amer. Math. Soc. 126 (1998), 2895-2902.

[8] E. T. Whittaker and G. N. Watson, A Course of Modern Analysis, 4th ed., Cambridge Univ. Press, 1966.

Lucent Technologies

1 Main St.

Cambridge, MA 02142-1517, U.S.A.

E-mail: seungson@lucent.com

Received on 21.7.1999

and in revised form on 19.6.2000 